\title{
Realistic Viewing and Manipulation of Radiographic Images on a Personal Computer-A User Interface for Educational Applications
}

\author{
Lincoln D. Stein, Jan Snydr-Michal, and Robert A. Greenes
}

\begin{abstract}
The authors have designed a user interface for the display and manipulation of radiographic images that is intended for use in digitized teaching files and computer-aided instruction (CAl) applications. By making the way the viewer interacts with a set of digitized images on the computer screen as similar as possible to the interaction with conventional films on a viewbox the authors have tried to create a system that combines the directness of the conventional teaching file with the power and flexibility of CAl. This is accomplished on readily available personal computer workstations.
\end{abstract}

Copyright $\odot 1991$ by W.B. Saunders Company

KEY WORDS: computers, education; computer-aided instruction; images, digitized; images, storage and retrieval; images, display.

$\mathbf{T}$ HE CONVENTIONAL film-based teaching file has long been a mainstay of radiology education for medical students and residents. As a teaching tool, it has the distinct advantages of simplicity and directness. Unlike textbooks and slide carousels, the teaching file is a direct link between the way the student examines and manipulates radiographic images during training and how he or she will do so during his or her professional career: the images appear on radiographic film and are manipulated by rearranging the films on a viewbox. The major disadvantages of the teaching file are the costs of accumulating and maintaining it, its physical bulk, the time it takes to find and pull a film jacket, and the limited ways in which the library can be indexed in order to identify and locate films of interest.

Recently there has been a great deal of interest in creating teaching files and film libraries that are linked to a computer data base. ${ }^{1,2}$ Under such a system the data base could be searched by criteria such as anatomic site, features, diagnoses, or imaging modality in order to quickly identify relevant radiographs. The simplest method of doing this is to use the computer to index a conventional teaching file so that films of interest can be located and pulled based on diagnosis or other features of interest. ${ }^{3,4}$ While this increases the flexibility of the teaching file, it does not overcome the problems of storage space and the delay involved in obtaining individual films. Alternatively, the computer data base can be used to reference film reproductions in a paper handbook or a slide collection. This approach sacrifices image quality for improved accessibility. A third approach stores images on videodisc and displays them under software control on a television screen. ${ }^{5}$ While dramatically speeding image access time and making it possible to create truly interactive film libraries, videodisc images are inferior to native film. They cannot be easily overlayed with graphics and text and do not lend themselves to grey-scale or magnification adjustments.

A fourth way to combine a radiographic images with a computer data base is to create a digitized image library in which images are stored in digital form on magnetic or optical disk where they can be accessed and displayed directly on the computer screen. This brings the potential capabilities of rapid access to large libraries of high-quality images without the disadvantages of the conventional teaching file.

Advantages of a library of digitized images over other approaches include high resolution, rapid image display, the ability to manipulate gray scale, availability of other image processing functions, and the ease of overlaying graphics and text on the images. Although still inferior to native film, the digitized image offers the highest image quality compared to the modalities discussed above. In addition a library made out of digitized images is easily duplicated, modified, and enlarged, and offers great flexibility.

From the Decisions Systems Group, Department of Radiology and Pathology, Brigham and Women's Hospital, Boston, $M A$.

Supported in part by Grants LM 04572 and LM 07037 from the National Library of Medicine, and CA 45574 from the National Cancer Institute.

Address reprint requests to Lincoln D. Stein, $M D, P h D$, Brigham and Women's Hospital, 75 Francis St, Boston, MA 02115 .

Copyright $\odot 1991$ by W.B. Saunders Company 0897-1889/91/0403-0005\$03.00/0 
Computer-aided tutorials, self-test assessments, clinical case studies, discussions, and pathological correlates can be built around any arbitrary subset of the digitized image collection. Examples of the use of digitized image libraries in diagnostic applications and teaching files are given in references 6 and 7. Disadvantages of digital images over videodisc relate to their larger storage requirements and the slower access times required to retrieve them.

The major tradeoff of the digitized image library versus conventional film is that the radiographs are displayed on a video display terminal (VDT) rather than in their native form. The nature of the interaction with the film is altered. Consider, for example, what happens when a radiologist examines a film: he or she gives the film a quick "once over" to obtain a general impression, then moves in to examine specific features more carefully. With mammograms, neonatal chest films, and certain bone films, it is usual to examine selected portions of the films with a magnifying lens to pick up fine features. The radiologist may then write on the film with a grease pencil to mark important features, or hold the film up to a bright light to illuminate overpenetrated areas. The nature of the interaction with an image presented on VDT is quite different. Indeed a recent reviewer of the subject of computers in radiology education conceded that "the process by which radiology is taught at the viewbox is difficult to duplicate on the computer."2

Since it is desirable to make the learning experience as close to actual practice as is feasible, the appearance, resolution, and manipulation of digitized teaching cases must be similar to the way the film images are seen in practice. Recently we have developed a user interface for image presentation that accomplishes this. Our guiding principles have been twofold. First, that the student's interaction with the image be as "true to life" as possible and simulate the manner in which real films are displayed and manipulated. Second, the system must display high quality images on a personal computer system equipped with modest amounts of memory but lacking specialized graphics hardware.

\section{MATERIALS AND METHODS}

Our software was written on a Macintosh II computer (Apple Computer, Cupertino, CA) equipped with 8 Mbyte of memory, an 80 Mbyte hard disk, and a 19" Radius color monitor (Radius, San Jose, CA), a $1280 \times 960 \times 8$ bit display. The minimum configuration required to run our software is 2 Mbyte of main memory, a color or gray-scale monitor capable of displaying 256 shades of color or gray, and a 20 Mbyte hard disk. Such a system is available for under $\$ 6,000$.

Images were digitized onto eight-track tape using a Konica Laser Scanner KFDR-S (Konica Corp, Tokyo, Japan), and downloaded to the Macintosh over Ethernet via a VAX-8600 minicomputer (Digital Equipment Corp, Marlboro, MA). Using this system, digitizations can be done at up to $85 \mu \mathrm{m}$ with up to 10 bits per pixel. Other digitizers produce 12 bit per pixel images, as do direct digital images such as computed tomography (CT), magnetic resonance imaging, and computed radiography images, which can also be transferred to the Macintosh.

The development system used was Macintosh Programming Workshop Assembly (Apple Computer, Cupertino, $\mathrm{CA}$ ), and Think Pascal (Symantec Corporation, Cupertino, CA). Think Pascal offers an object-oriented dialect of Pascal that provides powerful data hiding and abstraction facilities. The software was designed to be integrated with the Explorer- 2 knowledge management system developed by our laboratory, ${ }^{8.9}$ and its successor, the DeSyGNER system. ${ }^{10}$

\section{THE INTERFACE}

There are seven elements of our interface. The first is a virtual "viewbox" on which radiographic images are placed and positioned. Second are two tools for sizing and positioning the images. Third is a pair of controls for adjusting the contrast and brightness of individual images. Fourth is a "magnifying lens" that allows arbitrary areas of the radiographs to be enlarged. Fifth is a virtual "bright light" that allows the user to locally increase the brightness of the image to view overpenetrated areas. Sixth is a "grease pencil" that gives the user or author the ability to mark the radiograph with text and graphics. Finally, in addition to these elements are facilities for managing and linking text, graphics, and other didactic information to the images under the DeSyGNER knowledge management system.

\section{The "Viewbox"}

Figure 1 shows a display from our system running on the Macintosh II computer, a machine that is representative of the current upper end of personal computers. It uses a mouse pointing device to move a cursor across the screen, overlapping windows, and icon-based user commands.

In Fig 1, two digitized mammogram images have been placed next to one another in a single window that acts as a virtual "viewbox." As on a real viewbox, the images can be picked up and moved around to different positions. To do so, the user positions the mouse-controlled cursor on the image he wishes to move, depresses the mouse button, and drags the image to the desired position. Releasing the mouse button drops the image into place. This makes it easy 


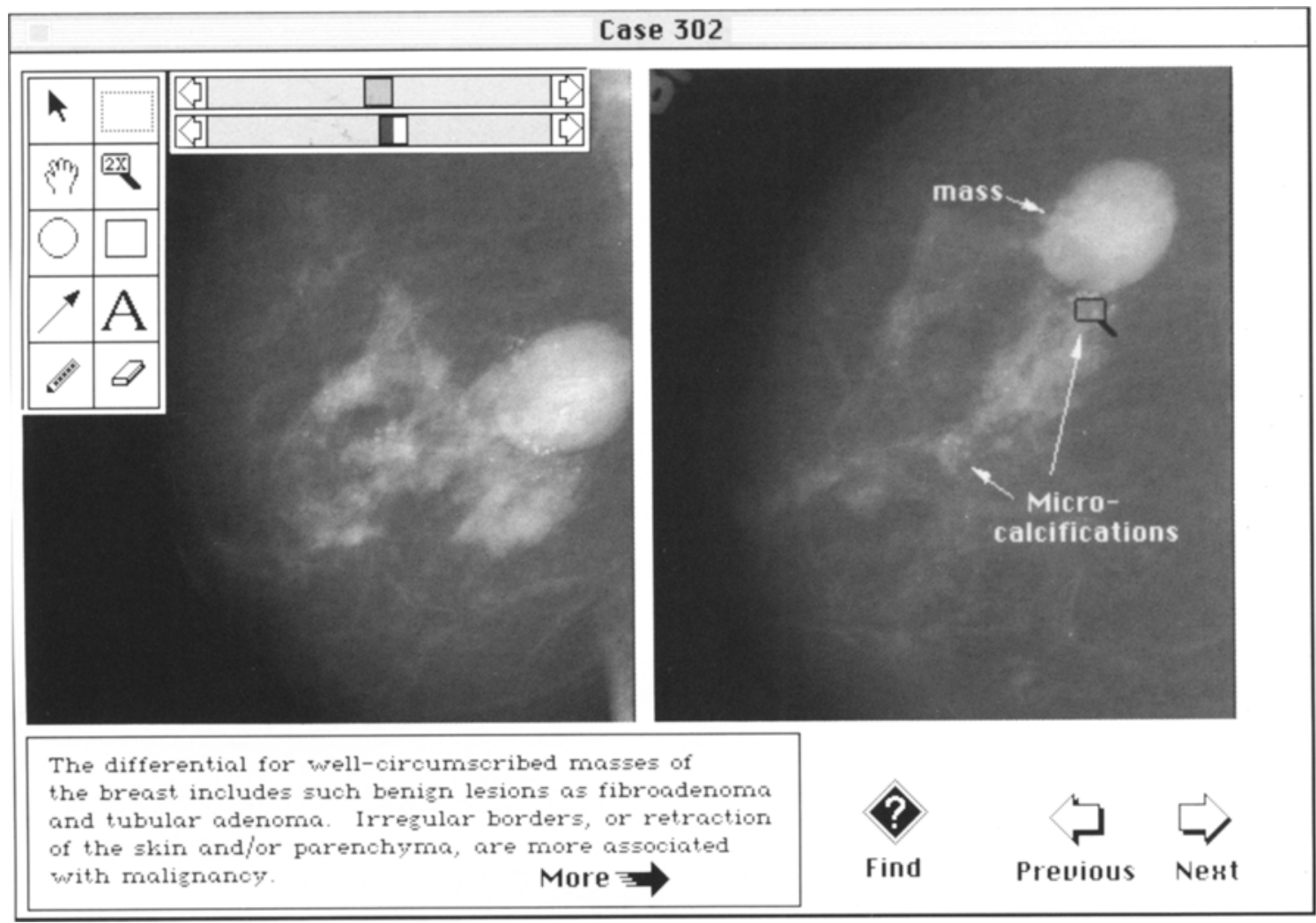

Fig 1. Elements of the user interface. The digitized images of two mammograms ( $A$ and $B$ ) are shown side by side in a single window. The images can be moved around, overlapped and resized with the mouse. Textual and graphical information can be added to the images as overlays. As described in the text, the two rectangular panels in the upper left of the leftmost image represent controls that allow the user to change the contrast and brightness of the image as well as to select various tools with which to manipulate the images. Text and graphics can be overlaid on the image, as shown at the right. Blocks of text and hypertext can be placed elsewhere within the window, or in other windows. A portion of a hypertext block can be seen underneath the leftmost image. In the example shown here, the user has positioned a small "magnifying lens" cursor over a portion of the image on the right that contains a feature of interest (calcifications), the magnification of which is shown in Fig 2 . (Reprinted with permission. ${ }^{10 a}$ )

to place posteroanterior and lateral films side by side or to arrange a series of films chronologically.

Two sets of controls are associated with each image; they appear to "float" over the image and can be moved about or made invisible at the user's discretion. These controls appear as the rectangular areas at the top and left margins of the left-most image in Fig 1. One set of controls in each image is the pair of horizontal arrow sliders shown at the top of the left-hand image used for contrast and brightness adjustment. The other set of controls is the group of symbols or "icons" along the left border of the image, which defines a set of tools that allows the user to manipulate the image in several ways. To select a tool, the user positions the cursor above the desired tool and clicks on it with the mouse. These controls will be described in the following sections.

\section{Sizing and Positioning}

Irrelevant portions of the images can be arbitrarily and reversibly cropped off by an intuitive maneuver. To remove part of the upper portion of an image, for example, the user selects the "arrow" tool from the left control panel, then moves the pointer to one of the top corners of the image, depresses the mouse button, and drags the corner downward to a new position. The portion of the image between the old and the new corner positions disappears. However, it can be restored simply by reversing this procedure. In addition, selection of the "hand" tool from the control panel on the left allows an image to be moved around within its cropping margins. Thus, one can not only determine the size of the cropped image, but the position of the image content itself.

Together, these features afford a great deal of flexibility in arranging groups of images. For example, a chronological series of CT images can be temporarily cropped to show only the sella turcica, allowing the user to concentrate his or her attention on changes in this region. Images can also be placed so that they overlap, although in this case the topmost image obscures the underlying images. This allows more images to be placed on the viewbox than can fit, just as overlapping films can be placed on real viewboxes.

Once a group of images is placed together and arranged to the user's liking, the arrangement can be saved to disk. Since it is the identification and positioning of the image that is saved and not the images themselves, the specifica- 
tion of the arrangement takes up little space and therefore can be saved, for example, on the user's personal floppy disk. Furthermore, several windows, each containing one or more radiograph images, can be present on the screen at once, giving the user multiple viewbox panels with which to work.

\section{Brightness and Contrast Controls}

The two top horizontal slider controls graphically show the user the amount of contrast and brightness in the image and allow him or her to adjust these parameters as needed, changing the global appearance of the image. This ability is needed to compensate for the fundamental difference in luminance between the VDT and the viewbox by permitting the selective highlighting of specific image features and allowing the user to adjust for individual differences between monitors. When multiple images are displayed on the viewbox simultaneously, the brightness and contrast of each can be adjusted individually, allowing the viewer to compensate for differences in film technique.

A limitation of the Macintosh display, as with most VDTs (including those of CT display consoles), is that it can only depict 256 shades of gray simultaneously ( 8 bits of data). Digitized radiographic images, however, can use up to 4,096 gray levels ( 12 bits). When 10,11, or 12-bit digitized images are displayed, the brightness and contrast sliders map these gray levels to the 256 levels available for display. By manipulating the sliders, therefore, the user can view all the information in the original image.

\section{The "Magnifying Lens"}

One of the most important tools is the magnifying lens, represented iconically as a miniature hand-held lens. When the magnifying lens tool is selected, the on-screen cursor that is linked to movements of the mouse turns into a small picture of a magnifier (this cursor is present in the center of the left-hand image of Fig 1). The user positions the cursor over some point of the image and presses the button on the mouse. At the moment the mouse button is depressed, a rectangular window pops up over the area selected and displays a magnified view of the image. This pop-up magnifying window is shown in Fig 2. While the mouse button is depressed, the user can move the mouse in any direction and the window containing the magnified image will slide over the fixed unmagnified image exactly as if the user were holding a true magnifying lens to the image. When the user releases the mouse button, the magnified image instantly disappears. The rapidity of the appearance and disappear-

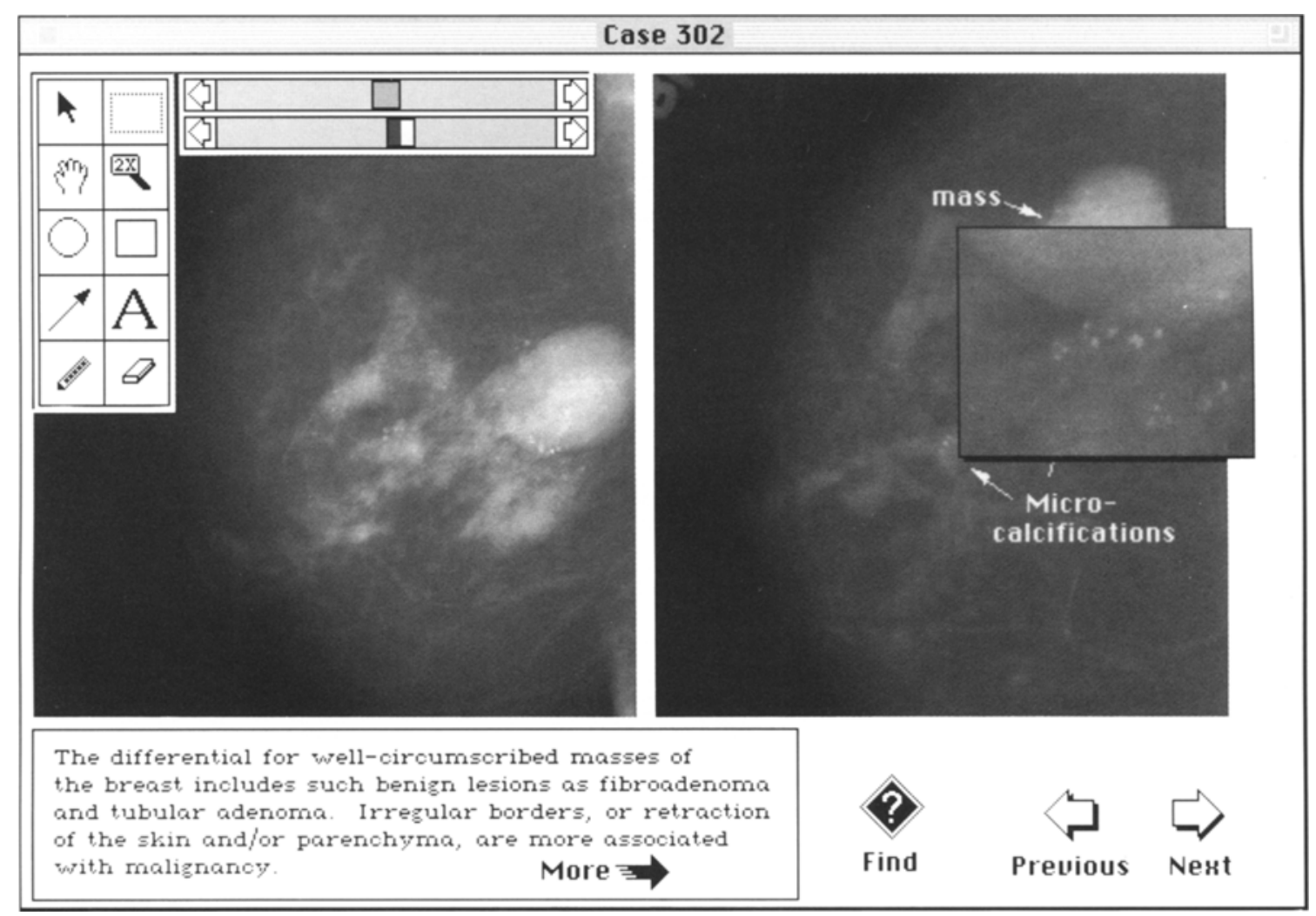

Fig 2. In this figure, the user has pressed the mouse button while the "magnifying lens" cursor was on the region shown in Fig 1. A rectangle has popped up showing greater detail in the region the user selected. As the mouse is moved around, this rectangle slides over the image, magnifying the portion beneath it. The user can change the degree of magnification so that even greater detail can be examined. By depressing a designated key, the user can also locally increase the brightness within the area of magnification, achieving a virtual "bright light" facility. (Reprinted with permission. ${ }^{10 a}$ ) 
ance of the magnifying window allows the user to examine multiple regions at high magnification without losing orientation and without performing a feat that would be "unnatural" with real film, as for example, making the entire image several times larger.

It is important to note that the magnified image produced by the magnifying lens tool represents true, full-resolution image data rather than false magnification through interpolation or replication of pixel values. For film images, this is because the data produced by commercial radiograph scanners have approximately fourfold greater resolution than can be displayed on the screen of a personal computer. The magnifying lens gives the user access to that highresolution data in a natural way. At highest magnification, approximately $90 \mu \mathrm{m}$ resolution is obtained, more than sufficient to show most fine diagnostic detail.

Although we wanted the magnifying lens tool to remain close to its real life model, we gave it certain capabilities that actual magnifying lenses do not have. The magnifying lens tool's magnification can be adjusted by the user by issuing various keyboard commands. The magnification is easily and almost instantly adjustable, allowing the user to scan through the image at low magnification and zoom in to high magnification to see very small details. The size and shape of the magnifying lens can also be adjusted to the user's preference.

As an alternative to the magnifying lens, the entire image can be displayed at higher magnification within the limits of the screen and moved around with the "hand" tool.

\section{The "Bright Light"}

Depressing a designated key on the keyboard puts the magnifying lens into "bright light" mode, a setting that increases the local contrast and brightness of the image shown within the lens. This approximates the effect of holding an overpenetrated section of the film up to a bright light in order to provide a virtual bright light facility without changing the overall brightness and contrast of the entire image. Like the magnifying lens itself, this technique allows the user to scrutinize an area of interest without changing the surrounding landmarks. The bright light mode can be applied while the magnifying lens is displaying the image at increased magnification. Alternately, the magnifying lens can be set to " $1 \mathrm{X}$ " (no magnification) so that the bright light facility can be used in isolation.

\section{The "Grease Pencil"}

Other icons represent a group of graphical tools that substitute for the grease pencil. Using these tools, the user can "mark up" the image in any arbitrary screen color, erase portions of the markings, and save and discard the set of markings to disk at his discretion. The markings are treated as a graphical overlay distinct from the actual image information, making it possible for different users to make different markings on the same image. Several tutorials that share the same image can each specify a different overlay for the image, emphasizing the features that are most relevant. Indeed, it is possible for users to save the overlays for a set of images on their personal floppy disks, creating in time a customized version of the film library.
Among the grease pencil tools is one that allows free hand markings to be made as well as an eraser for making free hand revisions. In addition to these, there are tools that allow users to draw circles, ovals, rectangles, lines, and arrows, as well as a tool to insert textual information such as captions and comments in any font size and style.

\section{Other Features}

While the ability to move, resize, and mark up images provides a degree of flexibility that is desirable in an unstructured teaching file, there are circumstances in which this much flexibility would be undesirable. For example, one anticipated use for this image viewing interface would be the creation of student tutorials, teaching files, and computer-driven textbooks. In these applications an author has typically already collected a set of radiographs and created captions, explanatory text, and figures to go along with the images. Under our system, the author positions the various graphic and text elements on the virtual viewbox in the desired configuration and then "locks" them in place so that they can be altered only temporarily or not at all. For example, the system could be set up so that the student can use the magnifying lens tool to examine the image and to make temporary markings with the crayon tool, but not have access to any of the other facilities.

Other functions allow the user to export and import all or a portion of the image to other Macintosh software. This feature allows marked-up radiograph images to be moved into manuscripts, tutorials, and other graphics manipulation packages. In addition, overlay graphics can be created in a foreign Macintosh graphics program and pasted over the radiographic image.

Eight-bit color images ( 256 colors) can be displayed side by side with greyscale images. The magnifying lens, grease pencil, and other tools work with color images as they do with the greyscale images with the exception of the bright light facility. This feature can be used for displaying radiological/pathological correlations.

The image viewing tools are designed for use within the DeSyGNER system, the Decision System Group's software environment for knowledge management, which is used for creating a variety of educational and decision support applications using hypermedia techniques. ${ }^{11}$ In this system, "page layout windows" such as those in Fig 1 can be created, and elements of the windows "hyperlinked" to other windows to facilitate browsing, exploration, structured tutorials, or question and answer sessions.

\section{RESULTS}

The major challenge in implementing our user interface was memory management. At highest resolution, a typical 8-bit grayscale image requires 2 to 4 Mbyte of storage. Ten- and 12-bit film images require more. The Macintosh II currently limits random access memory (RAM) to 8 Mbytes, and in practice much less memory is available when running multiple applications simultaneously. Our interface de- 
mands that multiple radiographic images be displayed side by side on the screen and that the full resolution data from any arbitrary portion of one of these images be instantly available if the user chooses to use the magnifying lens tool.

The first step in our solution to this problem was to preprocess the high-resolution scanned images into multiple smaller, subsampled views and to store the various resultant reductions together in the same disk file. This allows the small $1 \mathrm{X}$ images to be viewed without bringing the high-resolution images into memory. In most circumstances the user views and manipulates a subsampled image that is identical in dimensions to the original film. Only when the magnifying tool is utilized is the image displayed at full resolution.

The next step was to implement the virtual memory scheme shown in Fig 3. Each image, at each magnification available, is subdivided into multiple horizontal bands that are read in from disk to RAM when needed. When memory is scarce, unneeded portions of the images are purged out of RAM. A least-frequently-accessed algorithm is used to determine which portions of the images to purge. We chose to divide the images horizontally for two reasons. First, we have observed that most individuals scan horizontally across radiographs with a magnifying lens. By bringing the images into RAM as horizontal segments, we anticipate the user's actions. Second, horizontal segments are naturally stored as contiguous blocks on the disk. This minimizes the number of seeks the disk drive read head needs to make in order to read a segment, speeding disk access time.

A further refinement of this algorithm was to make use of additional RAM memory available from hardware add-on cards such as the $\mathrm{Na}$ tional Semiconductor NS8/16 board. The speed with which the RAM on these cards can be accessed is slower than main RAM but faster

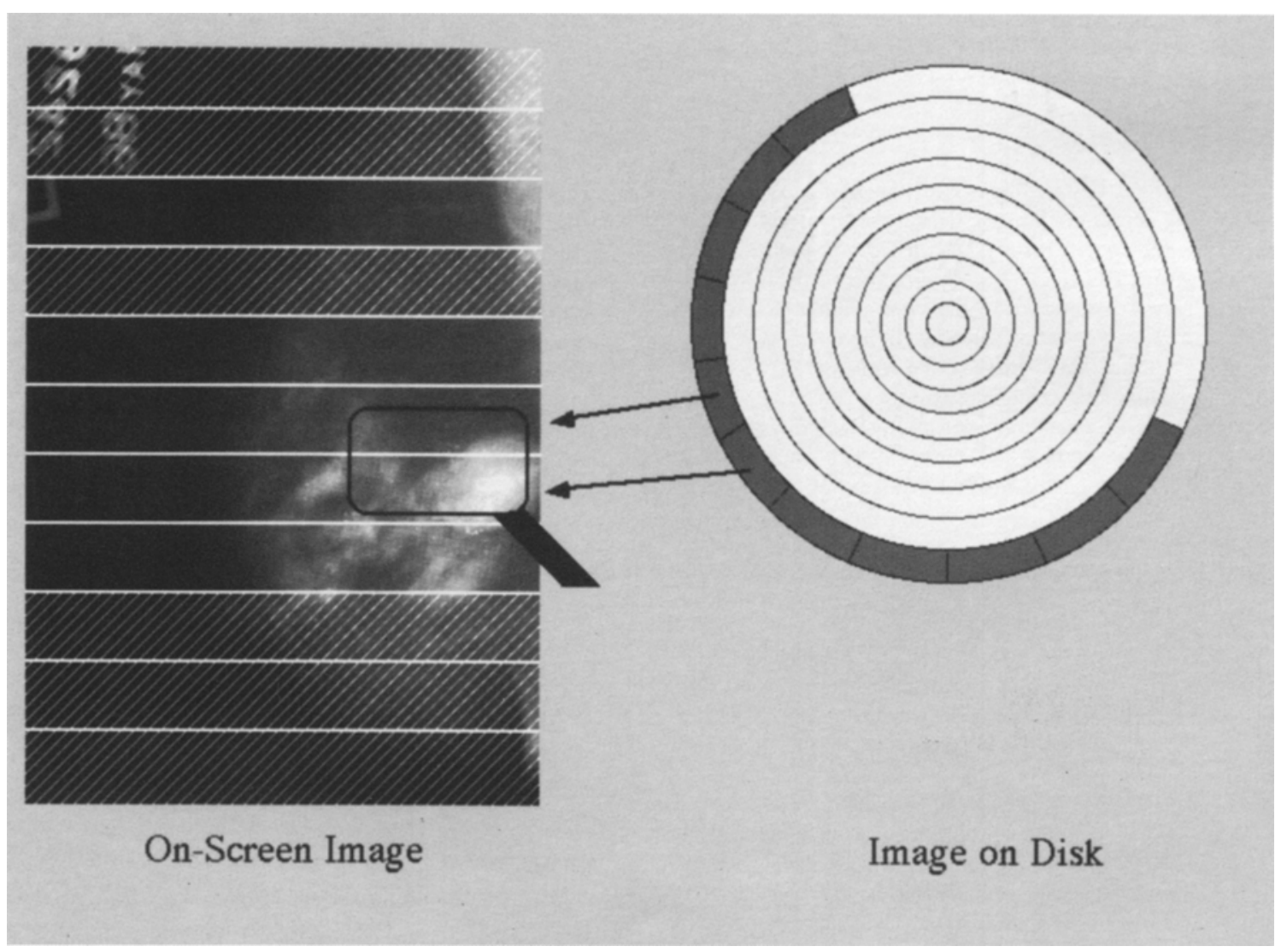

Fig 3. Virtual memory implementation scheme. The full-resolution image is subdivided into multiple horizontal bands stored sequentially on disk. As the user views different portions of the image with the magnifying glass, these portions are read from disk into memory. Portions of the image that have not been recently viewed are purged out of memory as necessary. 
than the hard disk. We implemented an optional caching scheme that goes into operation if the software senses that a RAM add-on board is present. Under this circumstance, unused image segments are cached in the RAM card so that the image data does not need to be read in from disk in many cases. This improves performance further.

While virtual memory solved the problem of limited RAM, it did not help reduce the large amount of hard disk storage space required for each image in all its magnifications. In order to reduce this problem, we store the images on disk in a packed run length limited (RLL) format. ${ }^{11}$ This format reduces the storage space required by average radiographs by $20 \%$ to $40 \%$ and has the advantage of having very rapid decompression times. Despite the necessity to decompress the data on the fly as it is read from disk, the performance losses are minimal. Other compressions schemes offer higher compression ratios but require more computation-intensive decompression. ${ }^{12}$ We are currently experimenting with several alternative algorithms.

On the Macintosh II running in a memory partition of 1.5 Mbytes and without an additional RAM card, the current implementation reads in from disk and displays a subsampled $500 \times 400 \times 8$ bit image in somewhat less than 2 seconds. The delay required to fetch overlayed graphics is negligible. Therefore a typical layout showing two $540 \times 350 \times 8$ bit images, such as those illustrated in the figures, is displayed in approximately 4 seconds. This time is reduced when the NS8/16 board is installed, or on Macintoshes with faster clock speeds, such as the IIci or IIsi. The two images shown in the figures require storage for three subsampled images each, the largest of which is $2150 \times$ $1400 \times 8$ pixels. Uncompressed, the two images require 7.6 Mbytes of total storage. With RLL compression, they consume 5.8 Mbytes, a savings of $26 \%$.

\section{DISCUSSION}

The digitized image library combines high quality images with the flexibility of computer aided instruction. Its advantages over other computer-based alternatives include the highest resolution, ease of duplication and distribution, and the ability to manipulate and enhance the images.

We have designed a user interface for the display and manipulation of digitized radiographic images that attempts to overcome the problem of the unnaturalness of the computerdisplayed image. By mimicking the way that people interact with film, the interface combines the power of the computer with the flexibility and naturalness of film reading. Although it is specifically designed to simulate film, the interface can also be applied to native digital modality images such as CT and MRI, since these are also usually reviewed on film.

Our interface displays digitized images that look and behave like film. The images can be moved about and overlapped on a virtual viewbox, marked on with a grease pencil and other graphic tools, selectively enlarged with a virtual magnifying lens to give the user access to the full resolution of the original radiograph without losing his global orientation, and selectively brightlit in areas of overpenetration. In addition, contrast and brightness controls allow the user to alter the image globally in order to highlight specific image features. It does this on a readily available personal computer running in a software environment specifically designed to produce computer-aided tutorials.

Computer-aided instruction applications are notorious for demanding a high degree of responsiveness. Although such judgements are necessarily subjective, we feel our system provides this response. At informal demonstration sessions, medical students, radiology residents, and practicing radiologists have all reacted favorably to the software interface as well as to its responsiveness. In assessing the specific performance measures given in the previous section it is important to note that the time to display images and the amount of storage they consume is dependent on the size and resolution required for the particular application. In many educational applications it may not be necessary to make use of the maximum resolution that can be obtained from the film scanner; it might be more appropriate instead to use several intermediate subsampled images for display. Under these circumstances the storage requirements would be much reduced. The software described here accepts arbitrary combi- 
nations of image dimension, resolution and pixel depth, allowing application authors great flexibility in making tradeoffs between storage consumption, display speed, and image quality.

The next steps in our project are the combination of the interface with a data base, and increasing its flexibility by allowing it to operate independently of the DeSyGNER environment. The combination is a cost-effective alternative to the conventional teaching file. In addition, because of its naturalness, we believe that it would be worthwhile to extend this interface to more powerful hardware to provide an environment in which to perform clinical diagnostic imaging.

\section{ACKNOWLEDGMENT}

We wish to acknowledge David Tarabar, Stephan Deibel, and Eric Slosser for valuable technical assistance in the implementation of this interface design.

\section{REFERENCES}

1. Sommer FG: Some applications for a personal computer data-base system in radiology. Am J Roentgenol 147:1075-1077, 1986

2. Tessler FN: Computer applications in radiology education: A challenge for the 1990s. Am J Roentgenol 152:11691172,1989

3. Sistrom CL, Black WC: A computerized filing system for radiology teaching cases. Am J Roentgenol 149:191-193, 1986

4. Garra BS, Kurtz D, Shawker TH, et al: A teaching file program for use in an ultrasound department. $J$ Ultrasound Med 5:313-317, 1986

5. Thursh DR, Mabry F, Levy A: Computers and videodiscs in pathology education: ECLIPS as an example of one approach. Human Path 17:216-218, 1986

6. Rowberg AH, Loop JW, Nelp WB: The design of a computer-assisted education system using a CT scanner computer. J Med Sys 8:111-114, 1984

7. Swett HA, Fisher PR, Cohn AI, et al: Expert systemcontrolled image display. Radiology 172:487-493, 1989
8. Greenes RA, Tarabar DB, Cope L, et al: Explorer-2: An object-oriented framework for knowledge management. Proceedings MEDINFO 1989. Amsterdam, The Netherlands, Elsevier Science, 1989 pp 29-33

9. Greenes RA, Tarabar DB, Krauss M, et al: Knowledge management as a decision support method: A diagnostic workup strategy application. Comp Biomed Res 22:113-135, 1989

10. Greenes RA, Deibel SRA: The DeSyGNER knowledge management architecture: A building block approach based on an extensible kernel. Artif Intell Med 3:95-111, 1991

10a. SCAR 90: Computer applications to assist radiology, in Arenson RL, Friedenberg RM (eds): Proc Computer Applications in Radiology, June, 1990

11. Pountain D: Focus on algorithms: Run-length encoding. Byte 12:317-320, 1987

12. Rabbani M, Jones PW: Image compression techniques for medical diagnostic imaging systems. J Digit Imag 4:65-78, 1991 